J Am Chem Soc. 2017 November 08; 139(44): 15738-15747. doi:10.1021/jacs.7b07507.

\title{
Ultrafast $\mathrm{CO}$ kinetics in heme proteins: adiabatic ligand binding and heavy atom tunneling
}

\author{
Abdelkrim Benabbas ${ }^{\dagger}$, Yuhan Sun ${ }^{\dagger}$, Thomas L. Poulos ${ }^{\ddagger}$, and Paul M. Champion ${ }^{\dagger, 2}$ \\ tDepartment of Physics and Center for Interdisciplinary Research on Complex Systems, \\ Northeastern University, Boston, Massachusetts 02115 \\ ‡Departments of Molecular Biology and Biochemistry, Chemistry, and Pharmaceutical Sciences \\ University of California, University of California, Irvine, CA 92697, USA
}

\section{Abstract}

We report on the ultrafast kinetics of $\mathrm{CO}$ rebinding to carbon monoxide oxidation activator protein (ChCooA) over a wide temperature range and make comparisons with the kinetics of $\mathrm{CO}$ and $\mathrm{NO}$ binding to protoheme (Fe protoporphyrin IX) and myoglobin (Mb). The CO binding to ChCooA is non-exponential over many decades in time at all temperatures studied, including room temperature. To describe this kinetic response we use a linear coupling model with a distribution of enthalpic rebinding barriers that is attributed primarily to protein-induced heterogeneity in the heme doming conformation (distributed barrier model, DBM). Above the solvent glass transition ( $\mathrm{T}_{\mathrm{g}} \sim 180 \mathrm{~K}$ ), CO rebinding kinetics displays an anti-Arrhenius behavior (i.e., the rate decreases as temperature increases) and this is ascribed to an evolution of the distribution toward increased heme doming and larger enthalpic barriers. Between $\mathrm{T}_{\mathrm{g}}$ and $\sim 60 \mathrm{~K}$, the non-exponential rebinding slows down as the temperature is lowered and the survival fraction follows the predictions expected for a quenched barrier distribution. However, below $\sim 60 \mathrm{~K}$ the rebinding kinetics do not continue to slow as predicted by the thermally activated DBM. A possible explanation for this behavior is explored that involves quantum mechanical tunneling of the iron atom along the heme doming coordinate. When the ultrafast $\mathrm{CO}$ rebinding kinetics of CooA are compared to the much slower $\mathrm{CO}$ rebinding in myoglobin, a two order-of-magnitude increase in the Arrhenius prefactor, from $\sim 10^{9} \mathrm{~s}^{-1}$ to $\sim 10^{11} \mathrm{~s}^{-1}$, is revealed. A similar prefactor is found for NO binding to CooA, which is typical for NO rebinding to ferrous heme systems. Because kinetic studies of $\mathrm{CO}$ rebinding to protoheme also reveal prefactors near $\sim 10^{11} \mathrm{~s}^{-1}$, we revisit the commonly held view that the $\mathrm{CO}$ binding reaction is non-adiabatic due to spin-forbidden $(\Delta \mathrm{S}=2)$ selection rules. A nonadiabatic sequential mechanism, involving first order $(\Delta S=1)$ transitions, is considered as a possible explanation for ultrafast $\mathrm{CO}$ rebinding; however, the relative crossing energies of the relevant spin states leads us to conclude that the $\mathrm{CO}$ ligand binding reaction is adiabatic rather than non-adiabatic and that entropic factors, rather than spin-selection rules, are the cause of the reduced Arrhenius prefactor for $\mathrm{CO}$ binding in $\mathrm{Mb}$ and $\mathrm{Hb}$.

\footnotetext{
${ }^{2}$ To whom correspondence should be addressed: Paul M. Champion, Professor Paul M. Champion, Department of Physics, 111 Dana Research Center, Northeastern University, Boston, MA 02115-5000, Phone: 617.373.2918, champ@ neu.edu.

Supporting information available

Details of CO binding kinetic analysis using the distributed coupling model; fits of the data at 300K; and a brief description of nonadiabatic atom transfer based on the theory developed by Jortner and Ulstrup. This material is available free of charge via the Internet at the ACS publications website.
} 


\section{Graphical Abstract}

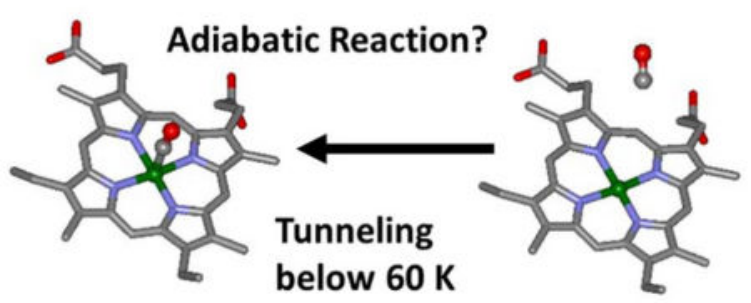

\section{Keywords}

heme proteins; transcription factors; ligand binding; kinetics; entropy; adiabatic reactions; tunneling

\section{Introduction}

Heme-based sensory proteins are an emerging class of proteins that perform key biological functions such as transcription regulation and production of second messengers, by selectively responding to changes in the levels of gaseous molecules such as $\mathrm{CO}, \mathrm{O}_{2}$ or $\mathrm{NO}^{1-2}$. One prototype of heme protein sensors is the carbon monoxide oxidation activator $(\mathrm{CooA})^{3}$. CooA from Carboxydothermus hydrogenoforms $(\mathrm{ChCooA})$ is a $\mathrm{CO}$-sensing transcription factor that regulates the expression of genes necessary for the bacterium to grow using $\mathrm{CO}$ as the sole energy and carbon source ${ }^{4-6}$. In addition to sensing, heme proteins can perform a variety of other biological functions including ligand transport, redox reactions and catalysis. Thus, the reactivity of the common heme prosthetic group must be tightly regulated and finely tuned by the structure of the surrounding protein and its dynamics. One of the methods that has been widely used to gain insights on the interplay between function, structure, and dynamics in heme proteins is the study of the rebinding kinetics and protein relaxation dynamics that follow ligand photo-dissociation by a short laser pulse ${ }^{6-26}$. The temperature dependent kinetics of $\mathrm{CO}$ rebinding to heme proteins, especially to myoglobin, has been extensively investigated over the years $7,9,11,13,18,22,25,27$. All of these previous investigations focused on analyzing $\mathrm{CO}$ rebinding kinetics over time windows that extend from $10^{-8}$ to $10^{3} \mathrm{~s}$. Up to now, detailed temperature dependent studies of $\mathrm{CO}$ rebinding kinetics in the sub-nanosecond time scale and over a broad range of temperature have only been conducted on heme model compounds ${ }^{20,28-29}$. However, there have been prior studies ${ }^{6,30-31}$ that have investigated ultrafast $\mathrm{CO}$ geminate recombination dynamics to heme proteins

These and other studies have shown that a wide variety of heme proteins, including CooA proteins ${ }^{6,32}$, nitrophorin $4^{21}, \mathrm{CBS}^{33}, \mathrm{tr}_{-} \mathrm{HBO}^{34}, \mathrm{RcoM}-2^{31}$ and mutants of cytochrome $\mathrm{c}^{35}$ rebind $\mathrm{CO}$ on the sub-nanosecond time scale with high geminate yield. In this report we explore the geminate rebinding kinetics of $\mathrm{CO}$ to $\mathrm{ChCooA}$, over a broad temperature range $(300 \mathrm{~K}$ to $20 \mathrm{~K})$. The biological relevance for the ultrafast $\mathrm{CO}$ rebinding and its high efficiency in CooA proteins is discussed in the context of the highly non-exponential kinetics and the enthalpic barrier distribution that develops very rapidly upon photolysis, and 
which is attributed to an ensemble of differing heme out-of-plane equilibrium positions. We observe that at very low temperatures ( $\$ 60 \mathrm{~K}), \mathrm{CO}$ rebinds to CooA significantly faster than expected, based on the quenched barrier distribution that is found at higher temperature. This suggests that tunneling along the heme doming coordinate may begin to assist the classical over-barrier reaction when the low temperature region is approached.

We also discuss the underlying reasons for the dramatic differences that are apparent when the $\mathrm{CO}-\mathrm{CooA}$ rebinding kinetics are compared to myoglobin $(\mathrm{Mb})$ and hemoglobin $(\mathrm{Hb})$. The large Arrhenius prefactors found for both $\mathrm{CO}$ and $\mathrm{NO}$ binding to CooA are considered in the context of the non-adiabatic spin selection rules that are often thought to dictate small molecule binding to ferrous heme proteins ${ }^{36-39}$. If the non-adiabatic $\mathrm{CO}$ binding model is invoked, the ultrafast heme-CO binding reaction for CooA must involve a sequential process, where $\Delta S=1$ transitions move the system rapidly from the $S=2$ iron spin state to the $S=1$ state and finally to the $S=0$ state. The much slower $C O$ rebinding reactions observed for $\mathrm{Mb}$ and $\mathrm{Hb}$ can then be explained by a direct $\Delta S=2$ transition having a much smaller (second order super-exchange) spin-orbit coupling with a maximum prefactor ${ }^{36}$ on the order of $\sim 10^{9}$. However, the sequential non-adiabatic process should reveal the presence of an intermediate $S=1$ iron spin-state, which has not been observed. The observation of ultrafast $\mathrm{CO}$ rebinding kinetics therefore suggests that the spin-orbit coupling is generally large enough that the reaction should be treated adiabatically. The large increase in the Arrhenius prefactor found for CooA compared to $\mathrm{Mb}$ would then be accounted for by a decrease in entropic barrier, rather than by an increased non-adiabatic matrix element.

\section{Results}

In Fig. 1 we show the rebinding kinetics of $\mathrm{CO}$ to $\mathrm{ChCooA}$ at temperatures above the solvent (glycerol/water) glass transition temperature $\left(\mathrm{T}_{\mathrm{g}} \sim 180 \mathrm{~K}\right)$. The $\mathrm{CO}$ geminate recombination occurs on the sub-nanosecond time scale and shows a highly non-exponential behavior. At $300 \mathrm{~K}$, more than $99 \%$ of the photolysed CO molecules rebind geminately. Because the escape fraction is so low, ChCooACO is an ideal system for studying the final step of $\mathrm{CO}$ binding to heme proteins ie: bond formation between $\mathrm{CO}$ and the iron. Figure 2 represents the kinetic traces of $\mathrm{CO}$ rebinding to ChCooA below $\mathrm{T}_{\mathrm{g}}$.

To fit the data presented in Figs. 1 and 2 we used a distributed linear coupling model for $\mathrm{CO}$ binding that has been described in detail elsewhere ${ }^{9,20}$ (see supplementary information, SI). In this model, the enthalpic barrier for $\mathrm{CO}$ binding is separated into two parts:

$$
H=H_{P}+H_{D}=\frac{1}{2} K a^{2}+H_{D}
$$

where $H_{P}$ represents the proximal barrier due to moving the heme from the domed to the planar configuration. In this expression, a represents the overall distance that the iron needs to move relative to the heme plane during the rebinding process and $K$ is an effective force constant between the iron-protein and the iron-porphyrin that describes the linear restoring forces. The quantity $H_{D}$ represents the remaining ( $a$-independent) contributions to the enthalpic barrier, which primarily involve ligand docking energy and other steric constraints 
associated with the distal pocket. The distribution of the iron-out-of plane displacements in the photolyzed ferrous state, $P(a)$, is taken to be a Gaussian with a mean value $a_{0}$ representing the average out-of-plane displacement and a variance $\sigma_{a}$ describing the width of the distribution. This leads to an asymmetric distribution of barrier heights, $g\left(H_{P}\right)$, for the proximal term that is given by Eq. S2 in the SI. The survival population of the fivecoordinate photoproduct at time $t$ after photolysis is then given by equation 2 ,

$$
N(t)=\int_{0}^{\infty} g\left(H_{P}\right) \exp \left(-k_{1} t e^{-\frac{H_{P}}{k_{B} T}}\right) d H_{P}
$$

With

$$
k_{1}=k_{0} e^{-\frac{H_{D}}{k_{B} T}}
$$

The quantity $k_{0}$ is the Arrhenius prefactor, and $k_{B}$ is Boltzmann's constant. The algebraic transformations performed on Eq. 2 to generate a simple 3-parameter non-exponential fitting function are given in the SI (Eq. S8). The black solid lines through the kinetic data in Figs. 1 and 2 are the fits to the kinetic data at each temperature. As can be seen from these two figures, this simple distributed barrier model accounts very well for the highly nonexponential rebinding kinetics of $\mathrm{CO}-\mathrm{ChCooA}$ over a wide range of temperatures. The data prior to $\sim 8$ ps were not considered in the fitting process because of the transient cooling signal that is mixed into the overall optical transmission signal, which is used to track the $\mathrm{CO}$ rebinding kinetics. The transient cooling signal becomes negligible within about $\sim 10 \mathrm{ps}$ following photon absorption.

Figure 3 displays the temperature dependent values of the mean iron out-of-plane displacement $a_{0}$ and its variance $\sigma_{a}$ for ChCooACO as extracted from the kinetic data (see SI-1 for details). The absolute values of these quantities depend on the effective force constant $K$, which is taken to be $22 \mathrm{~N} / \mathrm{m}$ (rather than $\sim 14 \mathrm{~N} / \mathrm{m}$ as found for $\mathrm{Mb}$ [??ref 9??] ) based on vibrational coherence measurements of ChCooACO where the frequency of the heme doming mode is upshifted relative to $\mathrm{Mb}^{40}$. For comparison, Fig. 3 also uses red symbols to indicate the temperature dependence of the equivalent parameters (i.e., $a_{0}, \sigma_{a}$ ) for the iron protoporphyrin IX (FePPIX) heme model system, $\mathrm{H}_{2} \mathrm{O}$-FePPIX-CO, which was investigated previously ${ }^{20}$. In Fig. 4 we plot $\log _{10} k_{1}$ (Eq. 3) as function of inverse temperature. The slope of this plot yields $H_{D} \sim 0$ and a prefactor $k_{0}=1.2 \times 10^{11} \mathrm{~s}^{-1}$. Figure 4 also displays the values of an average enthalpic barrier, $\langle H\rangle=\frac{1}{2} K a_{0}^{2}+H_{D}$, for $\mathrm{CO}$ binding to $\mathrm{ChCooA}$ as function of temperature above $\sim 60 \mathrm{~K}$. 


\section{Discussion}

\section{Heme structural evolution above $\mathbf{T}_{\mathbf{g}}$}

One important result of this study can be seen in Fig. 1. Above $\mathrm{T}_{\mathrm{g}}$, CO rebinding kinetics to ChCooA slows down as the temperature increases. This non-Arrhenius behavior is indicative of an increase of the average rebinding barrier as the temperature is raised in the region above $\mathrm{T}_{\mathrm{g}}$. Similar behavior has been reported previously for the kinetics of $\mathrm{CO}$ rebinding to the bare heme (FePPIX) and attributed ${ }^{20}$ to anharmonic temperature-dependent heme structural relaxation ${ }^{41}$ that favors a more out-of-plane geometry at elevated temperatures. The photolyzed heme evolution to a more out-of-plane geometry at higher temperature has also been directly correlated with the relaxation of the structure sensitive Band III of the heme group ${ }^{20}$.

Myoglobin ( $\mathrm{Mb}$ ) also shows a complicated anti-Arrhenius behavior for $\mathrm{CO}$ rebinding above the glass transition, but the $\mathrm{MbCO}$ kinetics take place on a much slower time scale $^{7,11-13,18,22}$. In principle, the slowing down of the CO rebinding kinetics in ChCooA (as the temperature is raised above $\mathrm{T}_{\mathrm{g}}$ ) could be due to relaxation of distal pocket residues as well as structural relaxation of the heme ${ }^{20}$. However, the distal contribution to the total rebinding barrier, carried by the term $H_{D}$, is very small $\left(H_{D} \sim 0\right.$, as shown in Fig. 4). This leads to the conclusion that the slowing down of the $\mathrm{CO}$ rebinding kinetics with increasing temperature in ChCooA must be primarily due to the relaxation of the heme geometry toward a more domed configuration as also observed for the "bare" heme (FePPIX) system ${ }^{20}$. The proximal barrier height distributions for $\mathrm{CO}$ rebinding, derived from the fitting parameters, are depicted in Fig. 5. Panel A of this figure clearly shows that the mean of the distribution is evolving toward higher energies as the temperature is raised; while panel B shows that the distribution is relatively constant between $80 \mathrm{~K}$ and $180 \mathrm{~K}$.

Non-exponential behavior and slowing down of ultrafast $\mathrm{CO}$ rebinding kinetics over a narrow range of increasing temperature has also been reported in other heme systems such as DNR, RrCooA and DosT ${ }^{30}$ as well as for RcoM- $2^{31}$. However, it has been suggested that the $\mathrm{CO}$ rebinding to these proteins involves no enthalpic barrier and the non-exponential behavior is attributed to a distribution of activation entropies ${ }^{30-31}$. In the case of RcoM- $2^{31}$, the anti-Arrhenius behavior was attributed to the slowing of protein motions as the temperature decreases, which might speed up $\mathrm{CO}$ rebinding by reducing the entropic barriers. However, this interpretation implies that below the glass transition, where the $\mathrm{CO}$ escape to the solvent is prevented and the protein motions are frozen, the $\mathrm{CO}$ geminate recombination rate should become independent of temperature. In contrast, the data in Fig. 2 and in ref ${ }^{20}$ show that $\mathrm{CO}$ rebinding kinetics to $\mathrm{ChCooA}$, as well as to the bare heme, slows down with decreasing temperature below $\mathrm{T}_{\mathrm{g}}$. This clearly demonstrates that $\mathrm{CO}$ geminate recombination in these heme systems involves a distribution of enthalpic, rather than entropic, barriers. Additionally, the fact that $\mathrm{CO}$ rebinding to FePPIX also shows a nonexponential kinetic response above glass transition ${ }^{20}$ strongly indicates that this nonexponential behavior is due to an ensemble of enthalpic barriers that originate from a distribution of heme configurations. 
The highly non-exponential kinetic response observed for $\mathrm{CO}$ rebinding to $\mathrm{ChCooA}$ above $\mathrm{T}_{\mathrm{g}}$ is attributed to rebinding of photolyzed $\mathrm{CO}$ molecules to an ensemble of hemes that are characterized by slightly different geometries that remain heterogeneous on the ultrafast time scale of the rebinding ${ }^{6}$. However, another possibility is that $\mathrm{CO}$ rebinding is affected by a "dynamic" relaxation of the heme or the protein, or that both barrier distributions and dynamic relaxation are involved. In the latter case, the rebinding barrier would be time dependent and vary on the same time scale as the kinetics. Such a relaxation process is better described using a stretched exponential ${ }^{1-12}$. In Fig. S1 of the SI, we compare the quality of fitting the $\mathrm{CO}$ rebinding kinetics of $\mathrm{ChCooA}$ at $300 \mathrm{~K}$ using both a stretched exponential function and the distributed coupling model ${ }^{9,20}$ described above. The stretched exponential fit fails to describe the long time tail of the kinetic response, whereas the distributed coupling model fits the data very well over the full time range. This is additional evidence that the non-exponential behavior of the kinetic response above $T_{g}$ arises primarily from an underlying barrier distribution that is well-described by the distributed coupling model.

These results also suggest that, even at room temperature, the photo-dissociated $\mathrm{CO}$ molecules in CooA face a "quenched" distribution of rebinding barriers resulting from a set of heme conformations that evolve slowly on the sub-ns time scale of $\mathrm{CO}$ rebinding. However, it is important to emphasize that, following CO photolysis above $\mathrm{T}_{\mathrm{g}}$, the entire protein is not necessarily frozen on the rebinding time scale. The data are consistent with residues of the protein undergoing conformational fluctuations on the picosecond and early nanosecond time scale so long as these fluctuations have a negligible effect on the equilibrium heme geometry. The larger scale conformational interconversions that affect the heme equilibrium geometry are expected to involve multiple residues and/or helices and are evidently taking place on timescales $\gtrsim 10 \mathrm{~ns}$.

\section{Role of entropy within the adiabatic reaction model}

Below $\mathrm{T}_{\mathrm{g}}$, the solvent is frozen and large conformational fluctuations of the heme and the protein are inhibited. The kinetic response of $\mathrm{CO}$ rebinding to $\mathrm{ChCooA}$ in this temperature range (Fig. 2), displays a normal Arrhenius-type behavior, where the rates increase with temperature. However, there is a non-exponential decay that is characteristic of a quenched distribution of rebinding barriers. As can be seen from Fig. 3, the values of both the first and second moments of the $P(a)$ distribution, describing the ensemble of heme conformations, are only weakly temperature dependent in the range from $180 \mathrm{~K}$ to $80 \mathrm{~K}$. This behavior is very similar to that of $\mathrm{CO}$ rebinding to the bare heme $\mathrm{e}^{20}$ and it also mimics the kinetics of $\mathrm{CO}$ binding to myoglobin, but on a much faster time scale ${ }^{7,9}$.

As mentioned above, the Arrhenius prefactor for $\mathrm{CO}$ rebinding to $\mathrm{ChCooA}$ is $k_{0}=1.2 \times$ $10^{11} \mathrm{~s}^{-1}$. Such a value of $k_{0}$ is very similar to what is found for $\mathrm{CO}$ binding to heme model compounds such as FePPIX, with and without a 2-methyl imidazole (2MeIm) heme ligand 20 (Table 1). Significantly, this is two orders of magnitude larger than the prefactor of $\mathrm{CO}$ binding to $\mathrm{Mb}\left(k_{0} \sim 10^{9} \mathrm{~s}^{-1}\right)^{7,9,12}$. Thus, within the adiabatic model for CO binding to heme, the value of $k_{0}$ can be related to the activation entropy, $\Delta S^{\dagger}$, for CO binding in the following simple way: 


$$
k_{0} \propto \nu_{0} \exp \left(\frac{\Delta S^{\dagger}}{k_{B}}\right)=\nu_{0} \frac{\Omega^{\dagger}}{\Omega_{0}}
$$

where $v_{0}$ is an attempt frequency. Thus, because the overall electronic spin state transition $(\Delta S=2)$ is the same for the $\mathrm{CO}$ binding reaction in CooA and $\mathrm{Mb}$, the large difference in the prefactor is explained by changes in either the number of states for the protein-ligand system in the unbound state $\left(\Omega_{0}\right)$, or in the rebinding transition state $\left(\Omega^{\dagger}\right)$, or both. In other words, the entropic barrier for $\mathrm{CO}$ rebinding appears to be much lower in ChCooA compared to $\mathrm{Mb}$. This indicates that the activation entropy can play an important role in controlling the $\mathrm{CO}$ rebinding rate to heme proteins and it can be tuned over wide range by adjusting the architecture of the protein.

When $\mathrm{CO}$ binds to ChCooA, it causes the displacement of the endogenous ligand (the Nterminal amino group) which triggers a set of protein motions that induce large conformational changes, especially in the DNA binding domain. This large protein conformational change accomplishes two important tasks. The first involves the rearrangement of the DNA binding domain into a narrow set of configurations that allows specific DNA recognition and binding $6,42-44$. The second is the formation of a tight hydrophobic trap for the bound $\mathrm{CO}$ so that the system remains in the active state long enough for transcription to take place ${ }^{6,42-44}$. Indeed, the crystal structure of LL-ChCooACO shows that the distal pocket of this protein is composed of a cluster of hydrophobic residues that surrounds the bound $\mathrm{CO}$, forming a tight cavity around the heme $\mathrm{e}^{5}$. We suggest that upon photo-dissociation, these residues keep the $\mathrm{CO}$ molecule in its upright orientation and greatly restrain its translational and rotational motions. This, along with the short time for CO binding, significantly reduces the entropic barrier that can build up and inhibit the ligand rebinding reaction. On the other hand, time resolved $\mathrm{IR}^{15}$ and the crystal structure of photolyzed $\mathrm{MbCO}^{45}$, show that the photolysed $\mathrm{CO}$ molecule lies approximately in the plane of the heme at a distance of $\sim 4 \AA$ away from the iron. The development of an entropic barrier takes place in $\mathrm{Mb}$ as the $\mathrm{CO}$ samples a large number of unbound conformations from which it must recover in order to find the correct upright transition state orientation from which it can rebind.

For physiological reasons, the distal pocket of myoglobin has been engineered to present a significant distal enthalpic barrier for $\mathrm{CO}$ rebinding (Table $\left.1, H_{D}=7 \mathrm{~kJ} / \mathrm{mol}\right)^{9}$. At physiological temperatures, the distal barrier helps to dramatically slow the initial rebinding so that a large entropic barrier also develops, which leads to a majority of the photolyzed CO molecules escaping into the solvent. Time resolved X-ray crystallography has shown that the distal E helix and the distal histidine, His64, move toward the location formerly occupied by the $\mathrm{CO}$ ligand within the first nanosecond following $\mathrm{CO}$ photolysis ${ }^{46}$. This forms a steric barrier for $\mathrm{CO}$ rebinding that is related to the large $H_{D}$ measured in $\mathrm{Mb}$. On the other hand, the present study has shown that a distal barrier in ChCooA is basically non-existent. This means that ChCooA has evolved a heme pocket that eliminates distal steric constraints and ligand docking sites that might retard the geminate recombination of $\mathrm{CO}$. The only barrier seen by $\mathrm{CO}$ is the intrinsic proximal barrier, $H_{P}$, which corresponds to the motion that brings 
the iron into the heme plane transition state where it vacates the $e_{g}$ orbitals so that the lowspin 6-coordinate $\mathrm{CO}$ bound state can be formed. This is highly relevant to the biological function of $\mathrm{ChCooA}$ as a transcription factor ${ }^{6}$. Indeed, upon $\mathrm{CO}$ binding, this protein has to undergo large conformational change from the inactive to the active state and it must stay in this state long enough to search and bind its target DNA; finally recruiting the RNA polymerase to start the transcription. To fulfill this requirement, the distal pocket in ChCooA has been engineered to form an efficient trap for $\mathrm{CO}$ so that, upon its thermal dissociation, $\mathrm{CO}$ undergoes very rapid geminate rebinding to the heme in order to preclude any reversal of the structural rearrangements that might lead to protein deactivation and the disruption of the transcription process $^{6}$.

The large reduction in the activation entropy for $\mathrm{CO}$ binding to ChCooA compared to $\mathrm{Mb}$ may be related to the fact that the entropic barrier can have synergistic dependence on the enthalpic barrier for ligand binding. As shown in Table 1, the average activation enthalpy for ChCooA $\left(\langle\mathrm{H}\rangle=7.5 \mathrm{~kJ} / \mathrm{mol}\right.$ at $300 \mathrm{~K}$ and $\langle\mathrm{H}\rangle=3 \mathrm{~kJ} / \mathrm{mol}$ below $\left.\mathrm{T}_{\mathrm{g}}\right)$ is much smaller than that for $\mathrm{Mb}\left(\langle\mathrm{H}\rangle=18 \mathrm{~kJ} / \mathrm{mol}\right.$ at room $\mathrm{T}$ and $\langle\mathrm{H}\rangle=12 \mathrm{~kJ} / \mathrm{mol}$ below $\left.\mathrm{T}_{\mathrm{g}}\right)$. This greatly speeds up $\mathrm{CO}$ rebinding in $\mathrm{ChCooA}$, which helps to prevent the full development of an entropic barrier. This occurs because the photolysed ChCooA+CO does not have time to fully sample all accessible states following dissociation. In such situations (i.e. when the rebinding time scale is less than the entropy production time scale), the "non-equilibrium" entropic barrier for $\mathrm{CO}$ rebinding can be significantly reduced ${ }^{20}$.

\section{Spin-orbit coupling and the non-adiabatic reaction model}

An alternative description of the prefactor becomes necessary if a non-adiabatic model is considered applicable to the CO-heme binding reaction. This model applies when friction is low and the spin-orbit coupling matrix elements, which couple transitions between different spin-states of the reacting system, are small relative to $k_{B} T$. A variety of quantum calculations have been performed on these systems in an attempt to discern the heme and ligand configurations that lead to the intersections of the electronic spin-states where the transitions occur ${ }^{36,38-39}$. The results depend somewhat on the basis set and functionals that are used ${ }^{38-39}$ and, therefore, it remains uncertain if the direct $\Delta S=2$ reaction must always be the dominant pathway for the $\mathrm{CO}$ binding reaction.

If the non-adiabatic reaction mechanism is to be generalized for $\mathrm{CO}$ binding, the protein architecture and heme structure must allow for an ultrafast reaction as observed for CooA. This is likely to involve sequential spin-allowed $(\Delta S=1)$ transitions where the iron $S=1$ spin state acts as an intermediate (see Fig. 6). On the other hand, as suggested by others $^{36-37,47-48}$, the much slower $\mathrm{CO}$ rebinding observed for $\mathrm{Mb}$ and $\mathrm{Hb}$ involves a "direct" $\Delta S=2$ transition (??shown in Fig. 6C) with a small (second order) spin-orbit coupling matrix element, which significantly reduces the prefactor relative to spin-allowed matrix elements. We also note that within the sequential model for ultrafast CO-heme binding, the barrier between the iron triplet state and the final singlet state must be small, as shown in Fig. 6A, in order to account for the absence of any detectible optical intermediate other than the quintet and singlet states. A small or non-existent barrier between the triplet and singlet states ensures that the transient population of the triplet state is essentially 
unobservable and that the rate limiting step only depends on the initial first order $(\Delta S=1)$ quintet-triplet iron transition.

However, recent quantum chemical calculations have carefully delineated the minimum energies for quintet-triplet and triplet-singlet crossing. The direct $(\Delta \mathrm{S}=2)$ quintet-singlet crossing appears to lie at the lowest energy and it has therefore been chosen as the likely reaction route for CO-heme binding in the non-adiabatic model ${ }^{37,39}$. This choice is also conveniently motivated by the small prefactors that are observed for $\mathrm{Mb}$ and $\mathrm{Hb}^{37}$, 49 . On the other hand, when the ultrafast heme-CO rebinding in CooA is considered within the nonadiabatic formalism, a sequential first order $(\Delta \mathrm{S}=1)$ transition must be invoked in order to increase the prefactor by two orders of magnitude.

Importantly, the triplet-singlet crossing appears at higher energy than the quintet-triplet crossing ${ }^{39}$ as depicted qualitatively in Fig. 6B, and this has important consequences for the sequential non-adiabatic model. Under this condition, the triplet state population should be a detectable intermediate during the ultrafast $\mathrm{CO}$ rebinding reaction. However, optical experiments ${ }^{30-31,34}$ reveal no evidence of the $S=1$ intermediate when the ultrafast rebinding is followed with broad spectral bandwidth. Only the reactant ( $\mathrm{S}=2$ quintet state) and the product ( $\mathrm{S}=0$ singlet state) along with a clear isosbestic point have been observed ${ }^{20}$. This fact, along with the relative spin-state crossing energies ${ }^{39}$, suggests that the non-adiabatic sequential model is not consistent with the observed properties of the ultrafast heme-CO rebinding reaction. Thus, the adiabatic model (discussed in the previous section) presents a much better explanation for the ultrafast $\mathrm{CO}$ rebinding that is observed in CooA and a variety of other heme systems, while still having the ability to account for the much slower rebinding kinetics observed in $\mathrm{Mb}$ and $\mathrm{Hb}$.

\section{Anomalously fast rebinding kinetics below $60 \mathrm{~K}$}

As can be seen from Fig. 3, the parameters that describe the distribution of heme geometries below $\mathrm{T}_{\mathrm{g}}$ (i.e., the average iron out of plane displacement, $a_{0}$, and its variance, $\sigma_{a}$ ) are nearly constant for both ChCooACO and $\mathrm{H}_{2} \mathrm{O}-\mathrm{FePPIXCO}{ }^{20}$ in the temperature range from $180 \mathrm{~K}$ to $80 \mathrm{~K}$. This suggests that at low temperature the distribution is "frozen in" and should remain nearly constant at lower temperatures. However, below $80 \mathrm{~K}$ the fits require distributions that have significantly smaller values of $a_{0}$ than expected. The average distribution for $\mathrm{CO}-\mathrm{ChCooA}$ in the range $80-180 \mathrm{~K}$ can be found from Fig. $5 \mathrm{~B}$ and this results in $\left\langle a_{0}\right\rangle=0.21 \AA$ and $\left\langle\sigma_{a}\right\rangle=0.07 \AA$. This average distribution can be used to simulate the $\mathrm{T}<80 \mathrm{~K}$ kinetic response, resulting in the red lines shown in Fig. 2 (from bottom to top: $60 \mathrm{~K}, 40 \mathrm{~K}, 22 \mathrm{~K}$ ). These simulations show that the low temperature $\mathrm{CO}$ rebinding kinetics are significantly faster than expected for an over-barrier process using the quenched distribution that we normally expect to be frozen in below $180 \mathrm{~K}$.

\section{Photon heating should generate a full distribution even at low temperature}

One possible explanation for the faster than expected $\mathrm{CO}$ rebinding kinetics below $60 \mathrm{~K}$ is that the thermal excitations at these temperatures are insufficient to fully develop the iron out-of-plane distribution seen at higher temperatures. The heme doming frequency for a fully relaxed heme protein is typically found in the $40-50 \mathrm{~cm}^{-1}$ range $\mathrm{e}^{50}$ and is located at 50 
$\mathrm{cm}^{-1}$ for $\mathrm{CooA}^{40}$. This excitation energy $\left(50 \mathrm{~cm}^{-1} \sim 75 \mathrm{~K}\right)$ corresponds to the temperature where deviations from the expected kinetics begin to clearly appear. If thermal excitations of the doming mode are required to help move the protein into the different conformational states that ultimately determine the distribution of heme out-of-plane distances, then a reduced mean out-of-plane displacement may begin to appear at temperatures below the doming mode thermal excitation energy.

However, the out-of-plane distribution will also be affected by the excess energy of the photon that ruptures the bond between the ligand and the iron. During the first few picoseconds the local temperature of the heme is much higher than the temperature of the surroundings due to impulsive heating of the chromophore ${ }^{51}$. The photon-induced vibrational heating alters the heme optical properties and its cooling affects the transient absorption kinetic response on the sub-10 ps timescale. It is for this reason that we do not attempt to fit the rebinding kinetics on timescales below $8 \mathrm{ps}$. We conclude that transient heating of the heme is clearly taking place and this should act to generate very similar quenched distributions at all temperatures below $\mathrm{T}_{\mathrm{g}} \sim 180 \mathrm{~K}$.

\section{Quantum tunneling at low temperature within the adiabatic and non-adiabatic models}

When the photolyzed distibution of heme doming geometries, $P(a)$, is assumed to be independent of temperature in the $10-80 \mathrm{~K}$ range, there is a another possibility that explains the unexpectedly fast kinetics below $60 \mathrm{~K}$. The increase in the low temperature kinetics could arise from the existence of a quantum mechanical tunneling channel that begins to compete with the classical over-barrier process. Tunneling effects involving the $\mathrm{CO}$ molecule have been previously suggested to explain the rebinding kinetics of $\mathrm{Mb}$ and other heme proteins at temperatures below $25 \mathrm{~K}^{27,52}$. Similar effects have also been observed for water rebinding to heme ${ }^{53}$.

As a simple approximation, we can consider the overall $\mathrm{CO}$ tunneling process for a generalized heme protein to be a combination of $\mathrm{CO}$ tunneling through the distal barrier of height, $H_{D}$, along with the tunneling of the iron from its out-of-plane to its in-plane position through the proximal barrier, $H_{P}$. In principle, this process can either be concerted or sequential. In the case where the protein presents significant distal barrier for $\mathrm{CO}$ binding, such as in myoglobin, quantum mechanical effects in the kinetic response can be dominated by $\mathrm{CO}$ tunneling through the distal barrier ${ }^{52}$. On the other hand, for proteins like ChCooA or protoheme, where the distal barrier is very small or absent $\left(H_{D} \sim 0\right)$, the possibility of heme tunneling from an out-of-plane to an in-plane position can then be revealed at low temperature.

Jortner and Ulstrup developed a general theory of non-adiabatic atom transfer based on nonradiative transitions between vibronic states corresponding to two distinct electronic configurations representing a reactant and product ${ }^{48}$. They applied this theory to explain $\mathrm{CO}$ recombination kinetics to $\mathrm{Hb}$ at low temperatures where the process is governed by tunneling. The rate is expressed as the product of an electronic coupling term $V_{a b}$ between the electronic states " $a$ " and " $b$ " and a thermally averaged Franck-Condon vibrational overlap integral ${ }^{48}$. They adopted a harmonic approximation for the Fe motion, and assumed a single vibrational doming mode, characterized by two harmonic potentials of frequency $\omega$ 
in both states " $a$ " $(\mathrm{S}=2)$ and " $b$ " $(\mathrm{S}=0)$, where a direct $(\Delta S=2)$ process was assumed. It was found that this model adequately described the low $\mathrm{T}$ kinetics of $\mathrm{CO}$ binding to $\mathrm{Hb}^{48}$ (See S3 of SI for a summary of the Jortner and Ulstrup results).

As discussed above for the non-adiabatic sequential model of CO binding to CooA, there is a much larger first-order $(\Delta \mathrm{S}=1)$ electronic coupling term associated with $V_{a b}$, relative to the second-order super-exchange term that arises if $\Delta \mathrm{S}=2$ (see SI Eq. S12). Although tunneling from a more-domed to a less-domed heme conformation is possible at the quintet-triplet state crossing energy, the final triplet-singlet crossing takes place at higher energy ${ }^{39}$ (as depicted qualitatively in Fig. 6B). This non-adiabatic scenario is difficult to reconcile with the fact that the iron triplet state has not been detected in these reactions ${ }^{30-31,34}$.

On the other hand, if the electronic coupling is strong enough that the reaction becomes adiabatic there will effectively be little dependence on spin selection rules and this is relevant to a question that has been debated for many years. Namely, whether ligand binding (particularly $\mathrm{CO}$ binding) to heme proteins is an adiabatic or a non-adiabatic process $36-37,48,54$. The dilemma is that the total spin of the $\mathrm{CO}$ is zero. Therefore, during the geminate recombination, the $\mathrm{Fe}^{2+}(\mathrm{S}=2)+\mathrm{CO}(\mathrm{S}=0)$ system moves from the unbound electronic state $\left(\mathrm{S}_{\mathrm{T}}=2\right)$ where the heme is domed and the $\mathrm{CO}$ is docked in the distal pocket to the bound state $\left(\mathrm{S}_{\mathrm{T}}=0\right)$ with a planar heme. Thus, the binding process involves both nuclear motion and an electronic state change that cannot be obviously described as adiabatic in both the electronic and nuclear coordinates. In principle, for a direct transition, one has to account for the rate of spin state change, $\Delta \mathrm{S}_{\mathrm{T}}=2$, as dictated by the second order spin-orbit coupling matrix element $V_{a b}$ (Eq. S12).

However, the accumulated experimental data presented here indicate that ligand binding to heme proteins is insensitive to spin selection rules (e.g., the prefactor $k_{0} \sim 10^{11} \mathrm{~s}^{-1}$ is equivalent for both $\mathrm{NO}$ and $\mathrm{CO}$ rebinding in $\mathrm{CooA}$ ) and thus the reaction can be described as an adiabatic process with little dependence upon the electronic coupling matrix element. When $V_{a b} \neq 0$, the two diabatic energy surfaces " $a$ " $\left(\mathrm{S}_{\mathrm{T}}=0\right)$ and " $b$ " $\left(\mathrm{S}_{\mathrm{T}}=2\right)$ repel each at the crossing point, resulting in two adiabatic surfaces separated by $2\left|V_{a b}\right|$ as shown in Fig. 7. This means that there can be a well-defined transition state on the lower surface and, if 2| $V_{a b} \mid \gg k_{B} T$, the upper surface is inaccessible. When the reaction takes place exclusively on the lower surface, it can be described by standard transition state theory or Kramers theory 55 . In this case, once the lower adiabatic surface has been determined, the electronic nature of the two initial diabatic surfaces can be ignored: ie, the expression for the rate no longer depends on $V_{a b}$.

On the other hand, if $2\left|V_{a b}\right| \leq k_{B} T$, the situation becomes complex and the rate must be described using non-adiabatic theories such as the Jortner-Ulstrup theory ${ }^{48}$ or Landau-Zener theory ${ }^{36-37}$. In both of these theories, the rate, or more precisely, the prefactor $k_{0}$ is proportional to $\left|V_{a b}\right|^{2}$ and it becomes dependent on spin selection rules. The selection rules suggest that the second order $\left(\Delta \mathrm{S}_{\mathrm{T}}=2\right)$ spin transition for the CO binding reaction will reduce the prefactor by several orders of magnitude (e.g., to $k_{0} \sim 10^{9} \mathrm{~s}^{-1}$ ) compared to the first order $\left(\Delta \mathrm{S}_{\mathrm{T}}=1\right)$ spin allowed channel that is present, for example, in NO binding $\left(\mathrm{S}_{\mathrm{T}}=\right.$ $3 / 2$ to $\mathrm{S}_{\mathrm{T}}=1 / 2$ ) where $k_{0} \sim 10^{11} \mathrm{~s}^{-136}$. Therefore, to treat the ultrafast heme-CO rebinding 
within the non-adiabatic model, a sequential process must be invoked, where the initial quintet-triplet $(\Delta S=1)$ transition becomes rate limiting through a first-order matrix element, and where the second $\Delta S=1$ transition, from triplet to singlet, is nearly barrierless (cf. Fig. 6A). While the sequential non-adiabatic scenario is a possibility, calculations of the minimum energy crossing points for the iron spin states ${ }^{39}$ indicate that a transient triplet state population should appear during the $\mathrm{CO}$ binding process, in contrast to what is observed.

It is also noteworthy that a number of recent studies $6,20-21,33$ demonstrate $\mathrm{CO}$ binding kinetics that are very different from $\mathrm{Mb}$ and $\mathrm{Hb}$. In all of these studies, $\mathrm{CO}$ more typically binds to the heme with a prefactor of $k_{0} \sim 10^{11} \mathrm{~s}^{-1}$ (Table 1), which is similar to that of NO binding ${ }^{19}$. Importantly, this trend is also maintained within a single system, e.g., CooA, where both $\mathrm{NO}$ and $\mathrm{CO}$ rebinding kinetics have been studied and very similar prefactors $\left(k_{0}\right.$ $\sim 10^{11} \mathrm{~s}^{-1}$ ) are found ${ }^{6}$. Furthermore, studies of heme protein samples in strong magnetic fields showed that ligand binding kinetics are insensitive to the applied magnetic field ${ }^{56}$. These results lead generally to the conclusion that ligand binding to a heme system is very likely to be an adiabatic reaction, with a spin independent prefactor. Although not universally accepted, Frauenfelder and Wolynes reached a similar conclusion by invoking frictional effects and analyzing the rebinding kinetics of $\mathrm{CO}$ and $\mathrm{O}_{2}$ to $\mathrm{Mb}^{54}$.

Thus, if we proceed under the assumption that $\mathrm{CO}$ binding to heme is an adiabatic overbarrier process at high temperature, we can use the Bell tunneling model ${ }^{57}$ to estimate the crossover temperature $T_{0}$ at which the tunneling channel begins to compete with the classical over-barrier process. This model predicts that at high temperature the rate of $\mathrm{CO}$ binding to each heme within the ensemble is given by a classical Arrhenius expression:

$$
k_{c}=k_{0} \exp \left(-H / k_{B} T\right)
$$

where $H$ is the barrier height. At very low temperatures, the rate is dominated by tunneling and is given as:

$$
k_{t}=k_{0} \exp (-\pi a \sqrt{2 \mu H} / 2 \hbar) .
$$

Because there is no distal barrier $\left(H_{D}\right)$ for $\mathrm{CO}$ binding in the ChCooA and PPIX systems, the application of Eq. 1 effectively calculates the tunneling of the iron along the doming coordinate from the out-of-plane position to the planar configuration. We recall that $a$ is a measure of the out of plane distance and $\mu$ is the reduced mass of the nuclei associated with the doming mode (which involves the histidine, the iron, the porphyrin, and potentially some of the surrounding protein residues). At intermediate temperatures, the rate can be calculated numerically ${ }^{57}$; but to simplify the problem we will assume that the total rate at any temperature is the sum of the two rates $k=k_{c}+k_{t}$. The crossover temperature $\mathrm{T}_{0}$ at which both rates are equal is given as: 


$$
T_{0}=\frac{\hbar}{\pi a k_{B}} \sqrt{\frac{2 H}{\mu}}
$$

In the case of $\mathrm{ChCooA}$ and protoheme where $H_{P} \cong \frac{1}{2} K a^{2}$, Eq. 7 can be written as

$$
T_{0}=\frac{\hbar}{\pi k_{B}} \sqrt{\frac{K}{\mu}}=\frac{\hbar \omega}{\pi k_{B}}
$$

where it is important to note that the distributed parameter, $a$, associated with the heme outof-plane equilibrium position, drops out of the expression for $T_{0}$. This means that the entire distribution should have roughly the same cross-over temperature and this is what leads to the rapid onset of anomalously rapid kinetics near $\sim 60 \mathrm{~K}$.

The doming frequency of photolyzed CooA-CO at low temperature may be larger than its equilibrium value at room temperature as suggested by vibrational coherence studies of the photoproduct of myoglobin, usually referred to as $\mathrm{Mb}^{*}$. Although values of $\sim 40 \mathrm{~cm}^{-1}$ are found for the fully relaxed doming frequency in vibrational coherence experiments on deoxy $\mathrm{Mb}$ at room temperature, when the coherence immediately following photolysis is examined ${ }^{58}$ there is an additional strong coherence amplitude that appears near $\sim 80 \mathrm{~cm}^{-1}$. This higher frequency component has been attributed ${ }^{58}$ to the initial photoproduct, $\mathrm{Mb}^{*}$, where photolysis has taken place but the protein is not fully relaxed. The doming frequency becomes time-dependent under these highly non-equilibrium conditions and anharmonic Fourier components associated with the evolution $\left(\mathrm{Mb}^{*} \rightarrow \mathrm{Mb}\right)$ are observed ${ }^{58}$. At temperatures below $T_{g}$, the photolysis generates a species that is generally thought to mimic $\mathrm{Mb}^{*}$ (i.e., the protein is unable to relax and make the conformational rearrangements that fully accommodate the photolyzed heme). In $\mathrm{Mb}^{*}$ there are likely to be fewer of the surrounding nuclei participating in the iron doming motion and this acts to lower the reduced mass and increase the frequency both at short time and at low temperature. Thus, we suggest that the larger value for the doming frequency associated with $\mathrm{Mb}^{*}$ is also more appropriate at low temperature. Similarly, when the CooA-CO coherence spectrum at room temperature ${ }^{40}$ is carefully examined, in addition to the doming frequency attributed to the relaxed equilibrium geometry $\left(\sim 50 \mathrm{~cm}^{-1}\right)$, there is also a strong component at $85 \mathrm{~cm}^{-1}$ as well as a new shoulder at $\sim 100 \mathrm{~cm}^{-1}$ immediately following photolysis. By analogy with the $\mathrm{Mb}^{*}$ results, we therefore suggest that the doming mode frequency for the low temperature photoproduct of CooA falls in the region $\sim 85-100 \mathrm{~cm}^{-1}$.

Using Eq. 8 with $\hbar \omega \sim 100 \mathrm{~cm}^{-1}$, we estimate the crossover temperature $T_{0} \sim 45 \mathrm{~K}$ where the tunneling rate becomes comparable to the adiabatic barrier crossing rate. Based on this simple analysis, it seems that the contribution due to iron tunneling from the out-of-plane to the in-plane heme position cannot be neglected as temperatures begin to approach $\sim 45 \mathrm{~K}$. Although this is somewhat surprising, it helps to explain the unexpectedly large $\mathrm{CO}$ rebinding rates that are found below $60 \mathrm{~K}$ in the CooA system. 


\section{Conclusions}

In summary, we have investigated $\mathrm{CO}$ rebinding kinetics to ChCooA over a wide range of temperatures (300K to $20 \mathrm{~K}$ ) and made comparisons to $\mathrm{CO}$ and $\mathrm{NO}$ binding in myoglobin ${ }^{7,9,19}$ as well as heme model compounds ${ }^{20}$. We found that $\mathrm{CO}$ binding to $\mathrm{ChCooA}$ is nonexponential at all temperatures and over many decades in time. To analyze the kinetic data we used a linear distributed coupling model with a distribution of enthalpic (rather than entropic) barriers that are effectively "frozen out" on time scales faster than the sub-ns CO geminate recombination. This barrier distribution is attributed to a heterogeneous ensemble of heme doming conformations. The main conclusions of this work are: (i) Above $\mathrm{T}_{\mathrm{g}}$, CO rebinding kinetics to ChCooA shows an anti-Arrhenius (rate decrease with increasing T) behavior that we attribute to a relaxation of the heme distribution, $P(a)$, as the temperature is raised. (ii) In contrast to myoglobin, the heme environment in CooA proteins has been engineered to eliminate the enthalpic barriers associated with the distal pocket (e.g., steric constraints and docking sites) and this significantly speeds up CO recombination. (iii) Within the adiabatic model for binding, the activation entropy plays a major role in controlling $\mathrm{CO}$ binding to heme proteins and it can be tuned over a wide range by adjusting the architecture of the protein. (iv) Ligand binding to heme proteins is very likely to be an adiabatic reaction with a spin independent prefactor. However, many of the existing theoretical studies treat this reaction as a non-adiabatic process ${ }^{36-37,48}$ suggesting that additional theoretical investigations would be useful to fully understand the fundamental mechanism of the heme-ligand binding reaction. (v) Below $60 \mathrm{~K}$, it appears that tunneling of the iron along the doming coordinate can affect the kinetic response of $\mathrm{CO}$ binding in ChCooA. (vi) Although the lessons learned from the investigations of ligand binding to $\mathrm{Mb}$ significantly advanced our understanding of this elementary reaction, the Mb results should not be generalized to all heme proteins. This work suggests that myoglobin is an outlier, rather than a prototype, for ligand binding to heme and heme proteins.

\section{Materials and Methods}

The pump-probe setup used in this experiment is described in detail elsewhere ${ }^{6,21}$. The ferric ChCooA was dissolved in a mixture of $20 \% 50 \mathrm{mM}$ Tris- $\mathrm{HCl}$ buffer containing $6 \mathrm{mM}$ $\mathrm{CaCl}_{2}$ and $500 \mathrm{mM} \mathrm{KCl}$, and $80 \%$ glycerol (vol/vol, $\mathrm{pH} \mathrm{8.0).} \mathrm{The} \mathrm{solution} \mathrm{was} \mathrm{then}$ saturated with $\mathrm{CO}$, reduced with Na-dithionate and loaded anaerobically in a $1 \mathrm{~mm}$ pathlength home built gold plated copper cell with two PMMA windows. The cell was then mounted on closed cycle helium cryostat equipped with digital temperature controller. Flash photolysis experiments were performed by using two amplified and synchronized Ti:Sapphire laser systems. The sample was excited with a $405 \mathrm{~nm}$ pump and the change in transmission of the probe at $440 \mathrm{~nm}$ was detected by a photodiode connected to a lock-in amplifier. The time resolution of the system is $2.5 \mathrm{ps}$. Fast optical scanning across the sample was performed with an X-Y galvo scanner so that fresh sample was interrogated with each pulse pair.

\section{Supplementary Material}

Refer to Web version on PubMed Central for supplementary material. 


\section{Acknowledgments}

We thank Dr. Mandula Borjigin for the CooA samples used in this study. This work is supported by grants from the NSF (CHE-1243948) to P.M.C. and NIH 57353 to T.L.P.

\section{References}

1. Aono S. Metal-Containing Sensor Proteins Sensing Diatomic Gas Molecules. Dalton Trans. 2008:3137-3146. [PubMed: 18688409]

2. Gilles-Gonzalez MA, Gonzalez G. Heme-Based Sensors: Defining Characteristics, Recent Developments, and Regulatory Hypotheses. J Inorg Biochem. 2005; 99:1-22. [PubMed: 15598487]

3. Roberts GP, Kerby RL, Youn H, Conrad M. CooA, a Paradigm for Gas Sensing Regulatory Proteins. J Inorg Biochem. 2005; 99:280-292. [PubMed: 15598507]

4. Youn H, Kerby RL, Conrad M, Roberts GP. Functionally Critical Elements of CooA-Related CO Sensors. J Bacteriol. 2004; 186:1320-1329. [PubMed: 14973040]

5. Borjigin M, Li HY, Lanz ND, Kerby RL, Roberts GP, Poulos TL. Structure-Based Hypothesis on the Activation of the CO-Sensing Transcription Factor CooA. Acta Crystallogr D. 2007; 63:282-287. [PubMed: 17327664]

6. Benabbas A, Karunakaran V, Youn H, Poulos TL, Champion PM. Effect of DNA Binding on Geminate CO Recombination Kinetics in CO-Sensing Transcription Factor CooA. J Biol Chem. 2012; 287:21729-21740. [PubMed: 22544803]

7. Austin RH, Beeson KW, Eisenstein L, Frauenfelder H, Gunsalus IC. Dynamics of Ligand-Binding to Myoglobin. Biochemistry. 1975; 14:5355-5373. [PubMed: 1191643]

8. Petrich JW, Poyart C, Martin JL. Photophysics and Reactivity of Heme-Proteins: A Femtosecond Absorption Study of Hemoglobin, Myoglobin, and Protohem. Biochemistry. 1988; 27:4049-4060. [PubMed: 3415972]

9. Srajer V, Reinish L, Champion PM. Protein Fluctuations, Distributed Coupling, and the Binding of Ligands to Heme Proteins. J Am Chem Soc. 1988; 110:6656-6670.

10. Anfinrud PA, Han C, Hochstrasser RM. Direct Observations of Ligand Dynamics in Hemoglobin by Subpicosecond Infrared-Spectroscopy. Proc Natl Acad Sci USA. 1989; 86:8387-8391. [PubMed: 2554314]

11. Ansari A, Jones CM, Henry ER, Hofrichter J, Eaton WA. The Role of Solvent Viscosity in the Dynamics of Protein Conformational-Changes. Science. 1992; 256:1796-1798. [PubMed: 1615323]

12. Tian WD, Sage JT, Srajer V, Champion PM. Relaxation Dynamics of Myoglobin in Solution. Phys Rev Lett. 1992; 68:408-411. [PubMed: 10045884]

13. Ansari A, Jones CM, Henry ER, Hofrichter J, Eaton WA. Conformational Relaxation and LigandBinding in Myoglobin. Biochemistry. 1994; 33:5128-5145. [PubMed: 8172888]

14. Springer BA, Sligar SG, Olson JS, Phillips GN. Mechanisms of Ligand Recognition in Myoglobin. Chem Rev. 1994; 94:699-714.

15. Lim M, Jackson TA, Anfinrud PA. Binding of CO to Myoglobin from a Heme Pocket Docking Site to Form Nearly Linear Fe-C-O. Science. 1995; 269:962-966. [PubMed: 7638619]

16. Ostermann A, Waschipky R, Parak FG, Nienhaus GU. Ligand Binding and Conformational Motions in Myoglobin. Nature. 2000; 404:205-208. [PubMed: 10724176]

17. Schotte F, Lim MH, Jackson TA, Smirnov AV, Soman J, Olson JS, Phillips GN, Wulff M, Anfinrud PA. Watching a Protein as It Functions with 150-ps Time-Resolved X-Ray Crystallography. Science. 2003; 300:1944-1947. [PubMed: 12817148]

18. Levantino M, Cupane A, Zimanyi L, Ormos P. Different Relaxations in Myoglobin after Photolysis. Proc Natl Acad Sci USA. 2004; 101:14402-14407. [PubMed: 15385677]

19. Ionascu D, Gruia F, Ye X, Yu AC, Rosca F, Beck C, Demidov A, Olson JS, Champion PM. Temperature-Dependent Studies of NO Recombination to Heme and Heme Proteins. J Am Chem Soc. 2005; 127:16921-16934. [PubMed: 16316238] 
20. Ye X, Ionascu D, Gruia F, Yu A, Benabbas A, Champion PM. Temperature-Dependent Heme Kinetics with Nonexponential Binding and Barrier Relaxation in the Absence of Protein Conformational Substates. Proc Natl Acad Sci USA. 2007; 104:14682-14687. [PubMed: 17804802]

21. Benabbas A, Ye X, Kubo M, Zhang ZY, Maes EM, Montfort WR, Champion PM. Ultrafast Dynamics of Diatomic Ligand Binding to Nitrophorin 4. J Am Chem Soc. 2010; 132:2811-2820. [PubMed: 20121274]

22. Johnson JB, Lamb DC, Frauenfelder H, Muller JD, McMahon B, Nienhaus GU, Young RD. Ligand Binding to Heme Proteins. 6. Interconversion of Taxonomic Substates in Carbonmonoxymyoglobin. Biophys J. 1996; 71:1563-1573. [PubMed: 8874030]

23. Srajer V, Teng TY, Ursby T, Pradervand C, Ren Z, Adachi S, Schildkamp W, Bourgeois D, Wulff M, Moffat K. Photolysis of the Carbon Monoxide Complex of Myoglobin: Nanosecond TimeResolved Crystallography. Science. 1996; 274:1726-1729. [PubMed: 8939867]

24. Kruglik SG, Jasaitis A, Hola K, Yamashita T, Liebl U, Martin JL, Vos MH. Subpicosecond Oxygen Trapping in the Heme Pocket of the Oxygen Sensor Fixl Observed by Time-Resolved Resonance Raman Spectroscopy. Proc Natl Acad Sci USA. 2007; 104:7408-7413. [PubMed: 17446273]

25. Kriegl JM, Bhattacharyya AJ, Nienhaus K, Deng P, Minkow O, Nienhaus GU. Ligand Binding and Protein Dynamics in Neuroglobin. Proc Natl Acad Sci U S A. 2002; 99:7992-7. [PubMed: 12048231]

26. Ye X, Demidov A, Champion PM. Measurements of the Photodissociation Quantum Yields of Mbno and $\mathrm{MbO}_{2}$ and the Vibrational Relaxation of the Six-Coordinate Heme Species. J Am Chem Soc. 2002; 124:5914-5924. [PubMed: 12010067]

27. Alberding N, Austin RH, Beeson KW, Chan SS, Eisenstein L, Frauenfelder H, Nordlund TM. Tunneling in Ligand-Binding to Heme Proteins. Science. 1976; 192:1002-1004. [PubMed: 1273579]

28. Miers JB, Postlewaite JC, Zyung TH, Chen S, Roemig GR, Wen XN, Dlott DD, Szabo A. Diffusion Can Explain the Nonexponential Rebinding of Carbon-Monoxide to Protoheme. J Chem Phys. 1990; 93:8771-8776.

29. Miers JB, Postlewaite JC, Cowen BR, Roemig GR, Lee IYS, Dlott DD. Preexponential-Limited Solid-State Chemistry - Ultrafast Rebinding of a Heme Ligand Complex in a Glass or Protein Matrix. J Chem Phys. 1991; 94:1825-1836.

30. Lobato L, Bouzhir-Sima L, Yamashita T, Wilson MT, Vos MH, Liebl U. Dynamics of the HemeBinding Bacterial Gas-Sensing Dissimilative Nitrate Respiration Regulator (DNR) and Activation Barriers for Ligand Binding and Escape. J Biol Chem. 2014; 289:26514-26524. [PubMed: 25037216]

31. Bouzhir-Sima L, Motterlini R, Gross J, Vos MH, Liebl U. Unusual Dynamics of Ligand Binding to the Heme Domain of the Bacterial CO Sensor Protein RcoM-2. J Phys Chem B. 2016; 120:1068610694.

32. Rubtsov IV, Zhang TQ, Nakajima H, Aono S, Rubtsov GI, Kumazaki S, Yoshihara K. Conformational Dynamics of the Transcriptional Regulator CooA Protein Studied by Subpicosecond Mid-Infrared Vibrational Spectroscopy. J Am Chem Soc. 2001; 123:10056-10062. [PubMed: 11592884]

33. Karunakaran V, Benabbas A, Sun YH, Zhang ZY, Singh S, Banerjee R, Champion PM. Investigations of Low-Frequency Vibrational Dynamics and Ligand Binding Kinetics of Cystathionine Beta-Synthase. J Phys Chem B. 2010; 114:3294-3306. [PubMed: 20155941]

34. Jasaitis A, Ouellet H, Lambry JC, Martin JL, Friedman JM, Guertin M, Vos MH. Ultrafast HemeLigand Recombination in Truncated Hemoglobin HbO from Mycobacterium Tuberculosis: A Ligand Cage. Chem Phys. 2012; 396:10-16.

35. Silkstone G, Jasaitis A, Wilson MT, Vos MH. Ligand Dynamics in an Electron Transfer Protein. Picosecond Geminate Recombination of Carbon Monoxide to Heme in Mutant Forms of Cytochrome C. J Biol Chem. 2007; 282:1638-49. [PubMed: 17114183]

36. Franzen S. Spin-Dependent Mechanism for Diatomic Ligand Binding to Heme. Proc Natl Acad Sci U S A. 2002; 99:16754-9. [PubMed: 12477933] 
37. Harvey JN. Spin-Forbidden CO Ligand Recombination in Myoglobin. Faraday Discuss. 2004; 127:165-177. [PubMed: 15471345]

38. Harvey JN. DFT Computation of the Intrinsic Barrier to CO Geminate Recombination with Heme Compounds. J Am Chem Soc. 2000; 122:12401-12402.

39. Strickland N, Harvey JN. Spin-Forbidden Ligand Binding to the Ferrous-Heme Group: Ab Initio and DFT Studies. J Phys Chem B. 2007; 111:841-852. [PubMed: 17249828]

40. Karunakaran V, Benabbas A, Youn H, Champion PM. Vibrational Coherence Spectroscopy of the Heme Domain in the CO-Sensing Transcriptional Activator CooA. J Am Chem Soc. 2011; 133:18816-18827. [PubMed: 21961804]

41. Stavrov SS. Optical Absorption Band III of Deoxyheme Proteins as a Probe of Their Structure and Dynamics. Chem Phys. 2001; 271:145-154.

42. Lanzilotta WN, Schuller DJ, Thorsteinsson MV, Kerby RL, Roberts GP, Poulos TL. Structure of the CO Sensing Transcription Activator CooA. Nat Struct Biol. 2000; 7:876-880. [PubMed: 11017196]

43. Coyle CM, Puranik M, Youn H, Nielsen SB, Williams RD, Kerby RL, Roberts GP, Spiro TG. Activation Mechanism of the CO Sensor CooA - Mutational and Resonance Raman Spectroscopic Studies. J Biol Chem. 2003; 278:35384-35393. [PubMed: 12796503]

44. Kubo M, Inagaki S, Yoshioka S, Uchida T, Mizutani Y, Aono S, Kitagawa T. Evidence for Displacements of the C-Helix by CO Ligation and DNA Binding to CooA Revealed by UV Resonance Raman Spectroscopy. J Biol Chem. 2006; 281:11271-11278. [PubMed: 16439368]

45. Schlichting I, Berendzen J, Phillips GN, Sweet RM. Crystal-Structure of Photolyzed Carbonmonoxy-Myoglobin. Nature. 1994; 371:808-812. [PubMed: 7935843]

46. Srajer V, Ren Z, Teng TY, Schmidt M, Ursby T, Bourgeois D, Pradervand C, Schildkamp W, Wulff M, Moffat K. Protein Conformational Relaxation and Ligand Migration in Myoglobin: A Nanosecond to Millisecond Molecular Movie from Time-Resolved Laue X-Ray Diffraction. Biochemistry. 2001; 40:13802-13815. [PubMed: 11705369]

47. Redi MH, Gerstman BS, Hopfield JJ. Hemoglobin Carbon-Monoxide Binding Rate - LowTemperature Magneto-Optical Detection of Spin-Tunneling. Biophys J. 1981; 35:471-484. [PubMed: 6268215]

48. Jortner J, Ulstrup J. Dynamics of Non-Adiabatic Atom Transfer in Biological-Systems - CarbonMonoxide Binding to Hemoglobin. J Am Chem Soc. 1979; 101:3744-3754.

49. Harvey JN. Understanding the Kinetics of Spin-Forbidden Chemical Reactions. Phys Chem Chem Phys. 2007; 9:331-343. [PubMed: 17199148]

50. Rosca F, Kumar ATN, Ye X, Sjodin T, Demidov AA, Champion PM. Investigations of Coherent Vibrational Oscillations in Myoglobin. J Phys Chem A. 2000; 104:4280-4290.

51. Henry ER, Eaton WA, Hochstrasser RM. Molecular-Dynamics Simulations of Cooling in LaserExcited Heme-Proteins. Proc Natl Acad Sci U S A. 1986; 83:8982-8986. [PubMed: 3024159]

52. Alben JO, et al. Isotope Effect in Molecular Tunneling. Phys Rev Letts. 1980; 44:1157-1160.

53. Lamb DC, Kriegl J, Kastens K, Nienhaus GU. Quantum-Mechanical Tunneling of Water in Heme Proteins. J Phys Org Chem. 2000; 13:659-663.

54. Frauenfelder H, Wolynes PG. Rate Theories and Puzzles of Hemeprotein Kinetics. Science. 1985; 229:337-345. [PubMed: 4012322]

55. Hanggi P, Talkner P, Borkovec M. Reaction-Rate Theory - 50 Years after Kramers. Reviews of Modern Physics. 1990; 62:251-341.

56. Zhang ZY, Benabbas A, Ye X, Yu AC, Champion PM. Measurements of Heme Relaxation and Ligand Recombination in Strong Magnetic Fields. J Phys Chem B. 2009; 113:10923-10933. [PubMed: 19588986]

57. Limbach HH, Lopez JM, Kohen A. Arrhenius Curves of Hydrogen Transfers: Tunnel Effects, Isotope Effects and Effects of Pre-Equilibria. Philosophical Transactions of the Royal Society BBiological Sciences. 2006; 361:1399-1415.

58. Rosca F, et al. Investigations of Anharmonic Low-Frequency Oscillations in Heme Proteins. J Phys Chem A. 2002; 106:3540-3552. 


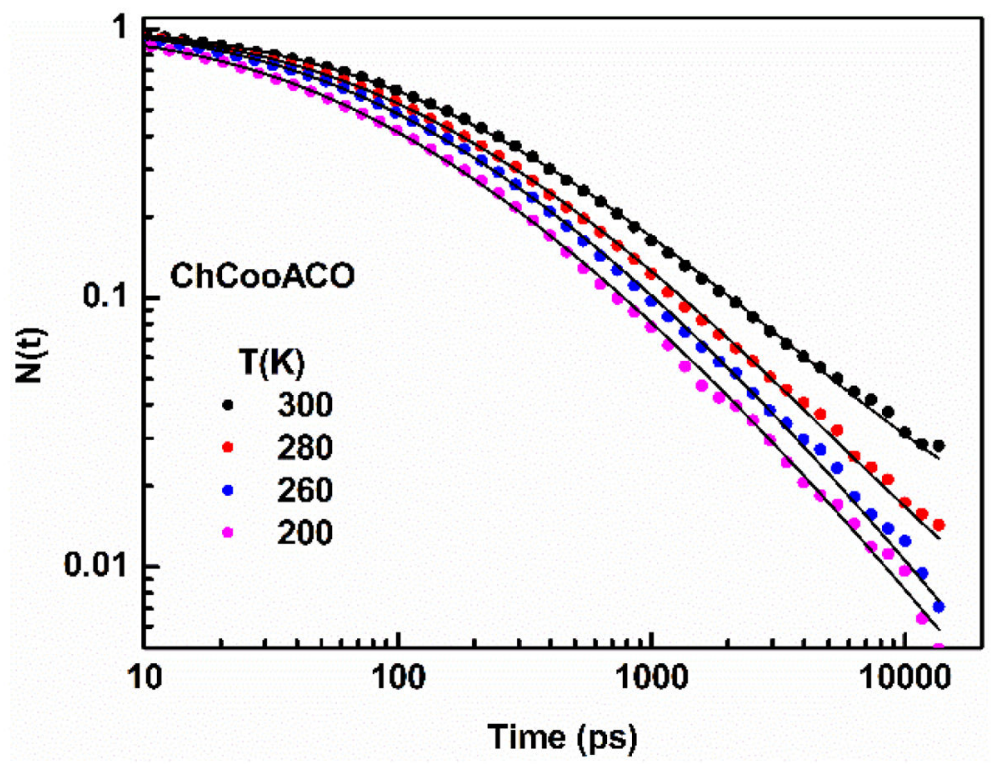

Figure 1.

$\mathrm{CO}$ rebinding kinetics to $\mathrm{ChCooA}$ above the glass transition temperature. The symbols represent the data and the solid lines represent the fits to the data using the linear coupling model. 


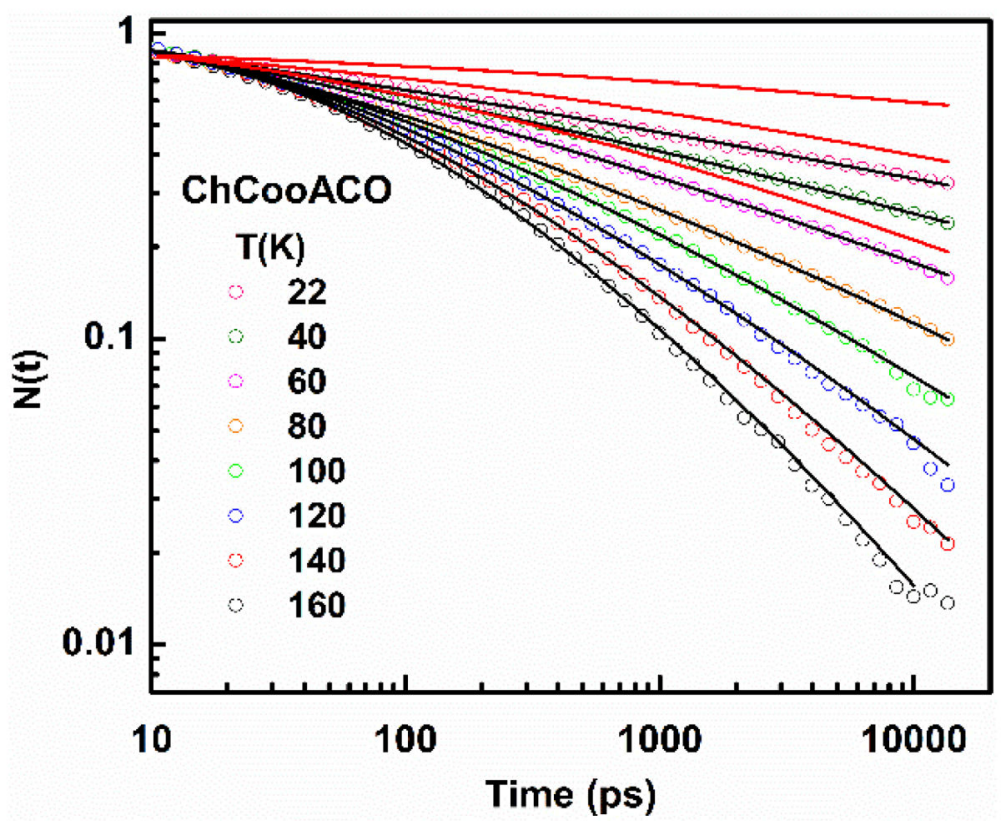

Figure 2.

$\mathrm{CO}$ rebinding kinetics to $\mathrm{ChCooA}$ below the glass transition temperature. The symbols represent the data. The black solid lines represent the fits to the data using the linear coupling model, without constraining the fitting parameters. The red lines represent the fits to the data at $\mathrm{T}=60 \mathrm{~K}, 40 \mathrm{~K}, 22 \mathrm{~K}$ respectively, using the linear coupling model with the expected quenched barrier distribution. For the quenched distribution calculations, the heme conformational distribution was frozen using the parameters found at $80 \mathrm{~K}: \mathrm{a}_{0}=0.2 \AA$ and $\sigma_{\mathrm{a}}$ $=0.07 \AA$. 


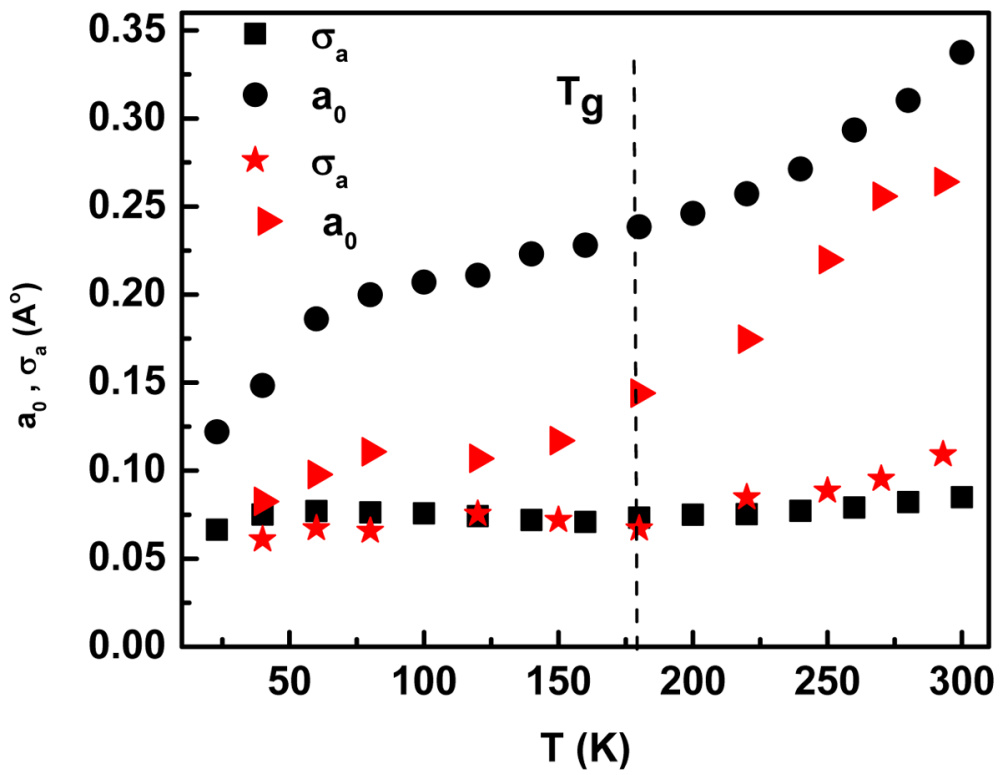

Figure 3.

Temperature dependence of the average iron out-of-plane displacement $a_{0}$ and its variance $\sigma_{a}$ as function of temperature for $\mathrm{CO}$ rebinding to ChCooA (black) and $\mathrm{H}_{2} \mathrm{O}-\mathrm{FePPIX}$ (red), as extracted from the kinetic data using the approach as outlined in the SI. 


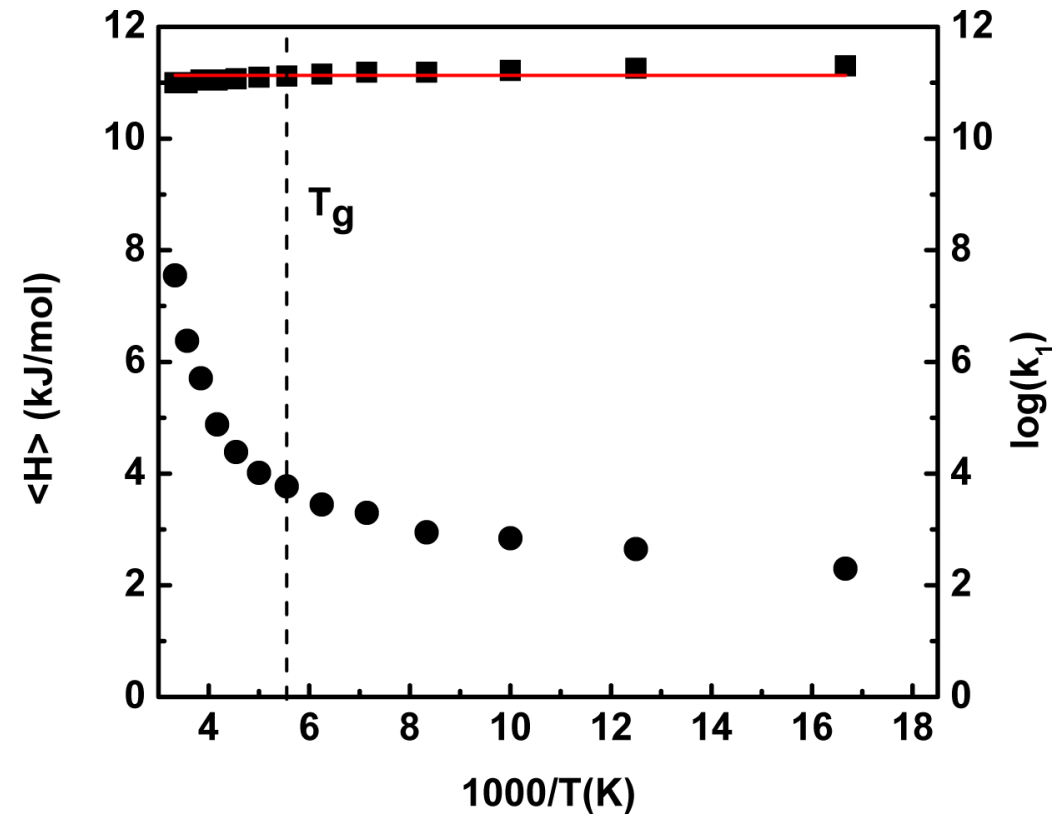

Figure 4.

Temperature dependence of the average enthalpic barrier $\left(<H>=\frac{1}{2} K a_{0}^{2}+H_{0}\right)$ for $\mathrm{CO}$ binding to ChCooA (circles), along with the parameter $\log \left(\mathrm{k}_{1}\right)$ (squares), where $\mathrm{k}_{1}$ is described in Eq 3. The temperature independent value of $\log \left(\mathrm{k}_{1}\right)$ gives $\mathrm{H}_{0} \sim 0 \mathrm{~kJ} / \mathrm{mol}$ and $\mathrm{k}_{0}$ $=1.2 \times 10^{11} \mathrm{~s}^{-1}$ 

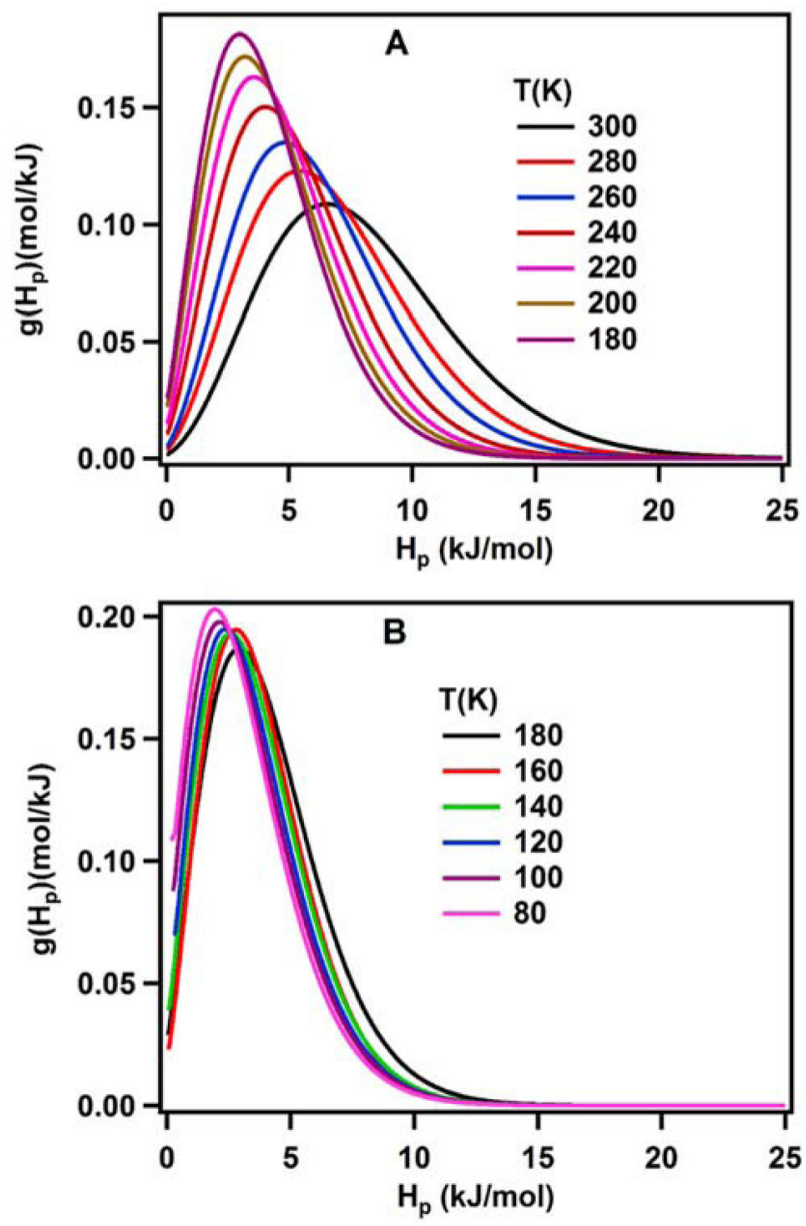

Figure 5.

Temperature dependence of heme proximal activation enthalpy probability distribution $g\left(H_{p}\right)$ for $\mathrm{CO}$ rebinding to ChCooA: $(\mathrm{A})$ above the glass transition temperature $\mathrm{T}_{\mathrm{g}} \sim 180 \mathrm{~K}$. (B) between 180 and $80 \mathrm{~K}$. 

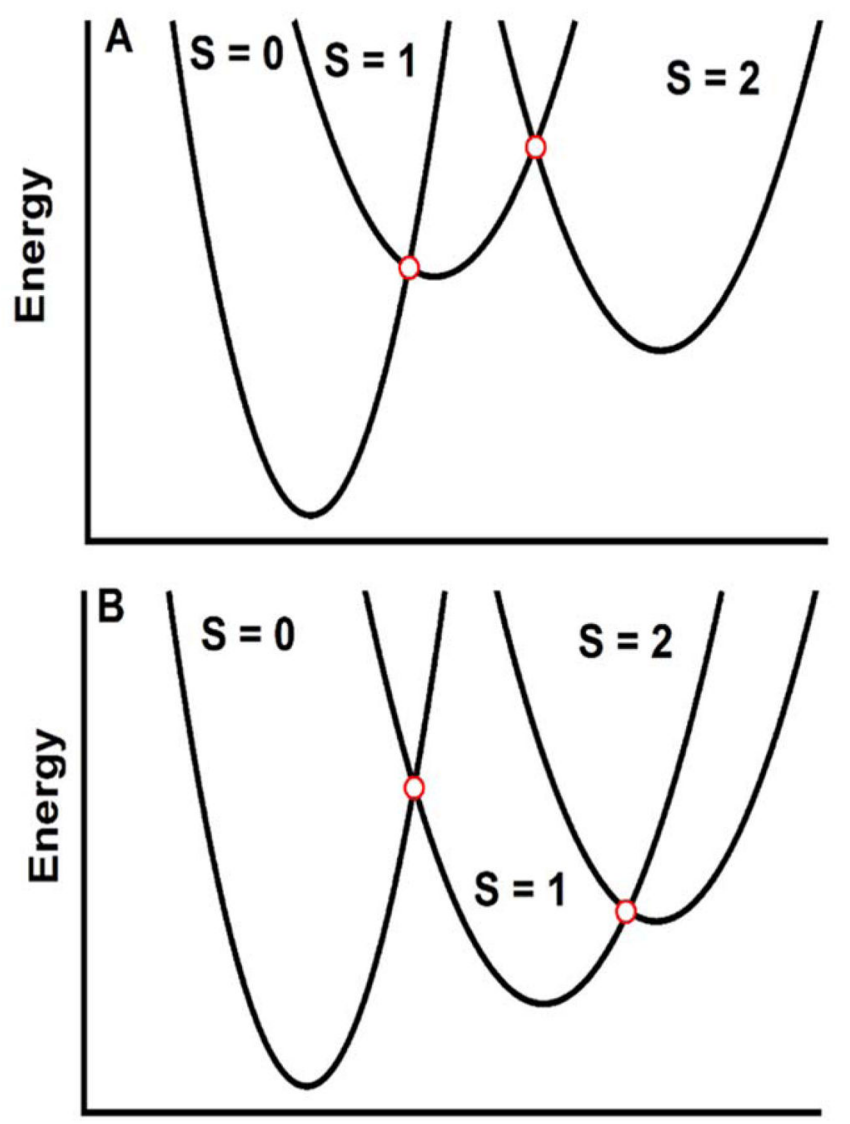

Doming Coordinate q

Figure 6.

Schematic representation of the electronic spin states for the heme $\mathrm{CO}$ system along the doming coordinate. (A) The barrier for the transition $2 \rightarrow 1$ is greater than that for $1 \rightarrow 0$ so that the first transition becomes rate limiting and no intermediate $S=1$ population can be detected; (B) The barrier for the transition $2 \rightarrow 1$ is smaller than that for $1 \rightarrow 0$ so that population in the $\mathrm{S}=1$ state should be detectable. 


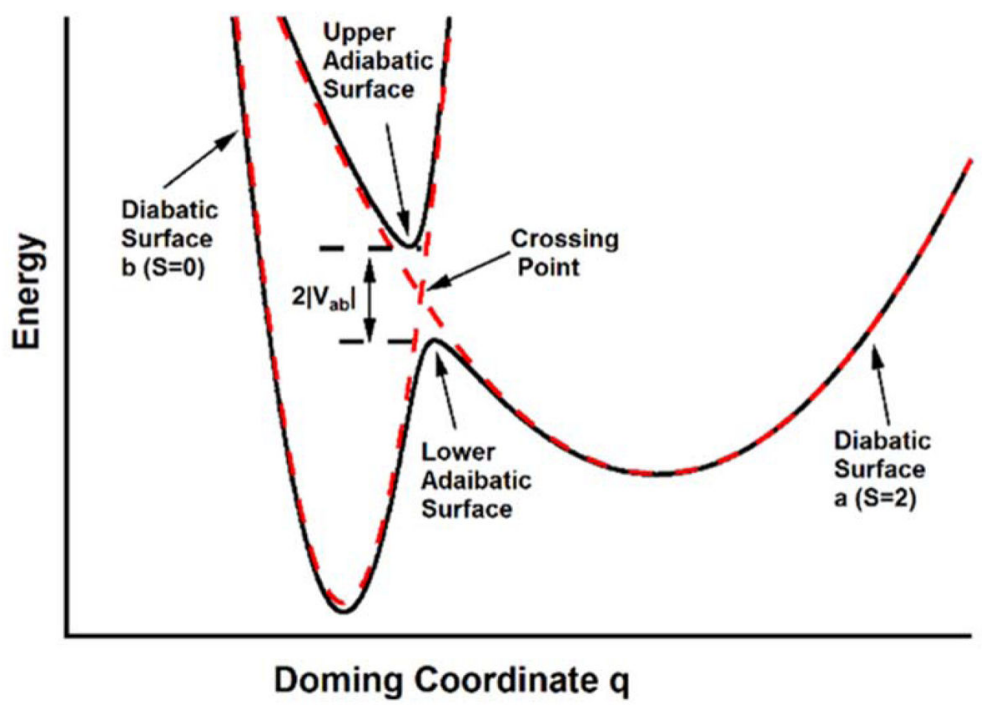

Figure 7.

Schematic representation of diabatic (dashed red) and adiabatic (black) potential energy surfaces for $\mathrm{CO}$ binding to the heme. 


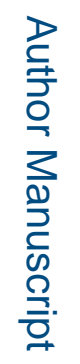

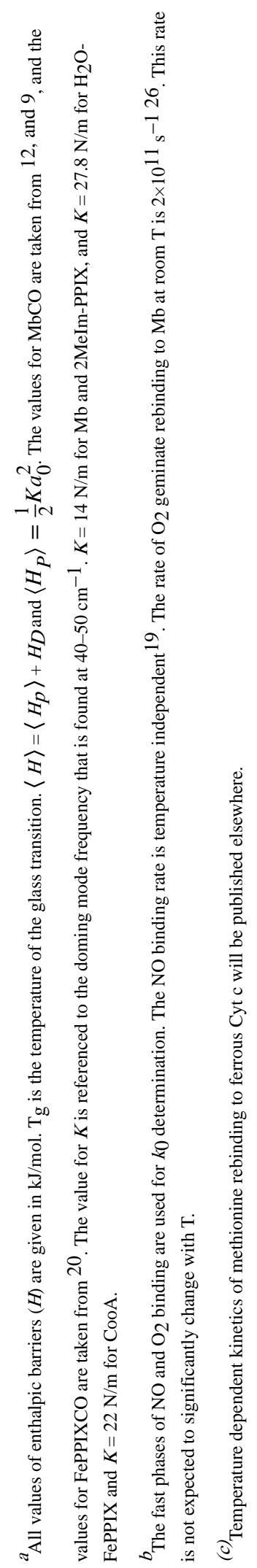

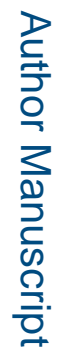



롤
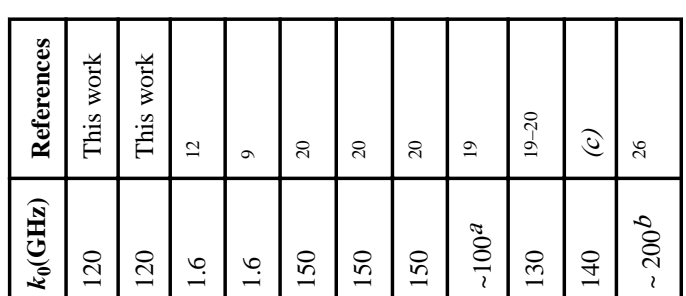

$\frac{0}{2}$

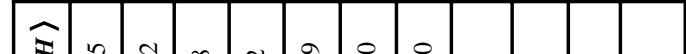

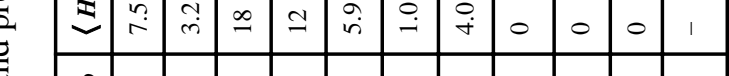

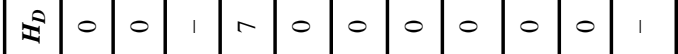

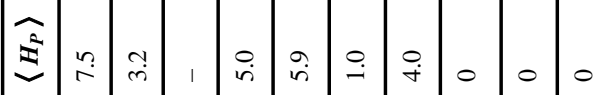

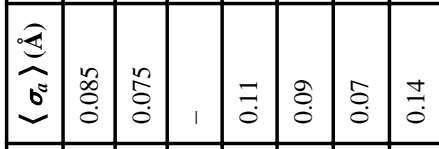

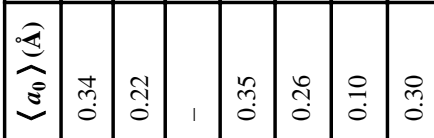

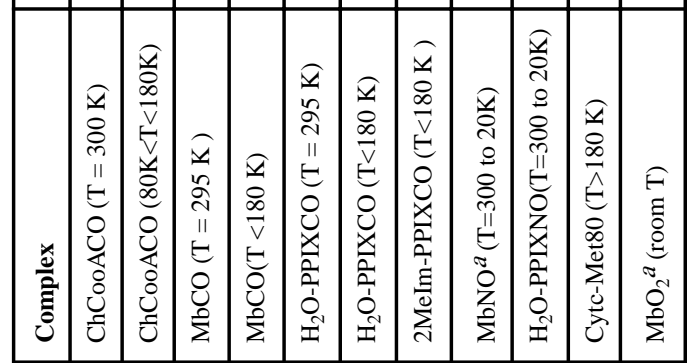

$\rightarrow$ 过 0 